\title{
Dupilumab-Associated Acquired Hemophilia A in an Elderly Male With Severe Eczema
}

\author{
Decerie B. Dayaga, b, Nasreen Shaikh ${ }^{\mathrm{a}}$, Patrick Lee ${ }^{\mathrm{a}}$, \\ Kenneth Granet ${ }^{\mathrm{a}}$
}

\begin{abstract}
This is a case of an 80-year-old Caucasian male with no personal or family history of coagulopathy, who was admitted for new onset painless hematuria followed by skin and significant deep soft tissue hemorrhage, with resultant anemia requiring blood transfusions. The hematuria started 1 week after he received an initial dose of subcutaneous injection of dupilumab for the treatment of refractory eczema. Few days later, he had spontaneous bruising on the tongue, anterior neck, right arm and entire left lower extremity in the absence of trauma and strenuous physical activity. His hemoglobin dropped to $7.2 \mathrm{~g} / \mathrm{dL}$ from a baseline of $15.0 \mathrm{~g} / \mathrm{dL}$. Laboratory tests revealed a prolonged partial thromboplastin time, reduced factor VIII activity, and presence of factor VIII inhibitors. Three units of packed red blood cells were transfused and oral prednisone $1 \mathrm{mg} / \mathrm{kg}$ daily was initiated. His hemoglobin stabilized. No further bleeding was noted and the ecchymoses gradually resolved.
\end{abstract}

Keywords: Acquired hemophilia; Hemophilia A; Dupilumab; Coagulopathy; Factor VIII inhibitor

\section{Introduction}

Dupilumab was approved by US Food and Drug Administration (FDA) in 2017 for the treatment of moderate to severe eczema. We present the first documented case of acquired hemophilia A after a single subcutaneous injection of dupilumab. Despite the proven safety and efficacy of this drug, physicians should be aware that acquired hemophilia A may be an associated adverse effect which may lead to life-threatening hemorrhage. More research is warranted to investigate this association.

Manuscript submitted July 11, 2019, accepted July 18, 2019

aDepartment of Internal Medicine, Monmouth Medical Center, Long Branch, NJ 07740, USA

${ }^{b}$ Corresponding Author: Decerie B. Dayag, Department of Internal Medicine, Monmouth Medical Center, Long Branch, NJ 07740, USA.

Email: deceriedayag@gmail.com

doi: https://doi.org/10.14740/jmc3336

\section{Case Report}

An 80-year-old Caucasian male with past medical history of hypothyroidism and severe generalized eczema and no personal or family history of bleeding diathesis was referred to our hospital for hematuria, generalized ecchymoses and anemia. Four weeks prior to admission, he received a subcutaneous injection of dupilumab $600 \mathrm{mg}$ for severe eczema that has been unresponsive to multiple courses of prednisone and methotrexate. One week after a single dose was administered, he developed painless gross hematuria for which he saw a urologist. Prior to cystoscopy, the urologist ordered outpatient blood tests which revealed a markedly elevated activated partial thromboplastin time of $127.3 \mathrm{~s}$ (normal: $30-42 \mathrm{~s}$ ). The patient was then referred to a hematologist for evaluation. Additional tests revealed a low factor VIII level of 4\% (normal 63-159\%), presence of factor VIII inhibitors, an elevated activated partial thromboplastin time (aPTT) that did not correct with a mixing study, and the absence of anticardiolipin antibodies. A few days later, the hematuria spontaneously resolved. The hematologist recommended that no treatment was necessary at that point because the hematuria had already resolved spontaneously. However, after a few days, the patient developed recurrent bruising over the tongue, anterior neck (Fig. 1), right arm and entire left lower extremity (Fig. 2). He was then sent to our hospital for evaluation of persistent bleeding and anemia.

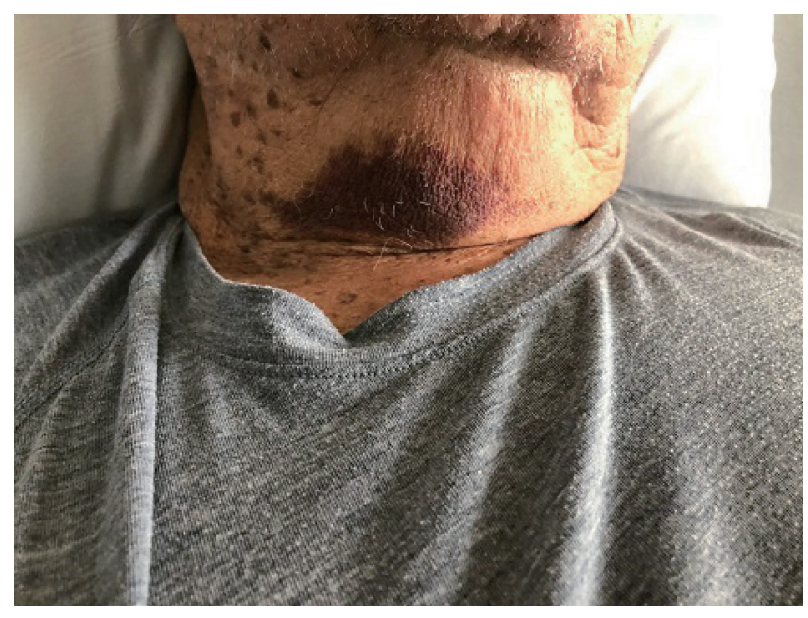

Figure 1. Skin and soft tissue bleeding on the anterior neck. 


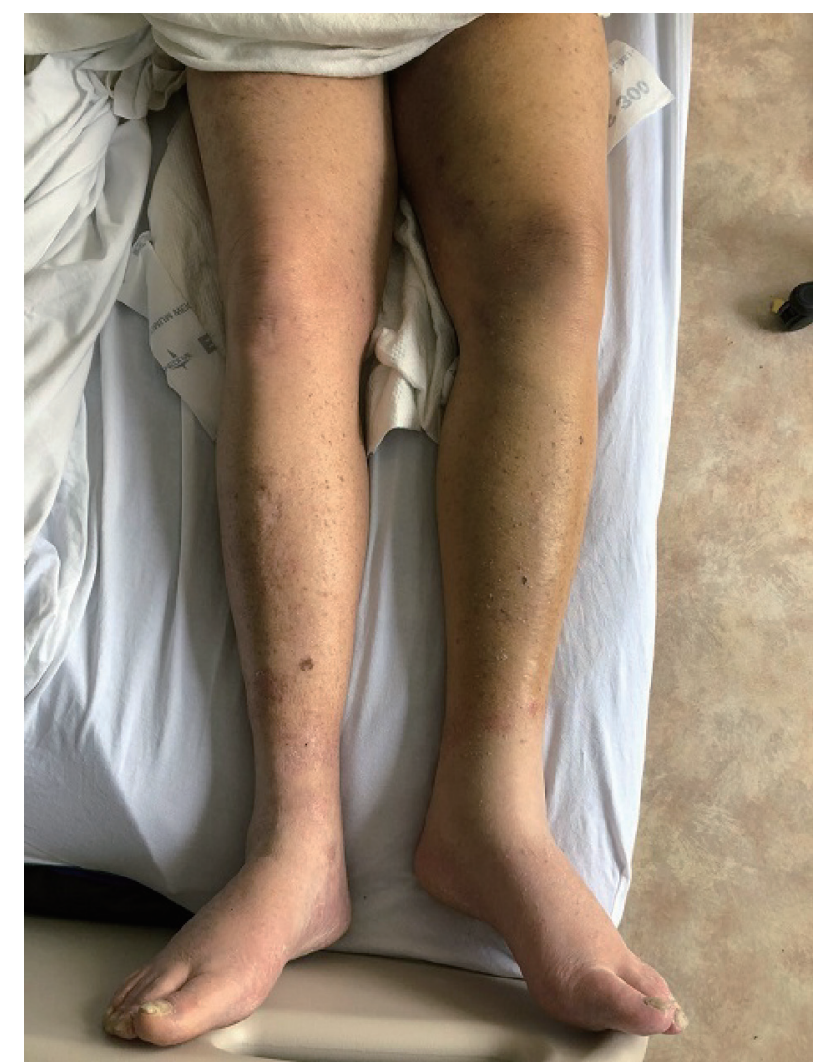

Figure 2. Noticeable ecchymosis and increased girth of the entire left lower extremity signifying deep tissue ecchymosis of the entire extremity.

\section{Investigations}

Laboratory test on admission showed a significant drop in hemoglobin of $7.2 \mathrm{~g} / \mathrm{dL}$ from a baseline of $15 \mathrm{~g} / \mathrm{dL}$, hematocrit $22.4 \%$, platelet count 242,000 , mean corpuscular volume (MCV) $97.1 \mathrm{fL}$, mean corpuscular hemoglobin (MCH) 31.2 pg and white blood cell count of $7,800 \times 10^{3} / \mu \mathrm{L}$ with normal differential. Detailed coagulation workup showed an isolated elevation in aPTT of $63.9 \mathrm{~s}$ (normal 24.1 - $31.2 \mathrm{~s}$ ) not corrected by a mixing study, normal prothrombin time of 10.6 $\mathrm{s}$ and international normalized ratio 1.0. Factor VIII activity was not quantifiable due to presence of factor VIII inhibitors at 74 Bethesda units (BU) detected 2 weeks prior to admission. Factor IX activity was normal. Anti-double stranded DNA antibody, antinuclear antibody, hepatitis panel and human immunodeficiency virus (HIV) antibody were negative. Renal and liver function tests were normal.

\section{Treatment}

During the hospital stay, his hemoglobin dropped to a nadir of $6.8 \mathrm{~g} / \mathrm{dL}$ then gradually improved to $9.1 \mathrm{~g} / \mathrm{dL}$ after receiving three units of packed red blood cells over $72 \mathrm{~h}$. He remained hemodynamically stable throughout the hospitalization. He was started on daily prednisone $1 \mathrm{mg} / \mathrm{kg}$ tablet and discharged to home with instructions to take prednisone for 21 days and follow up at the clinic.

\section{Outcome and follow-up}

One week after discharge, his aPTT improved to $40.7 \mathrm{~s}$ from $63.9 \mathrm{~s}$ while on oral prednisone $1 \mathrm{mg} / \mathrm{kg}$ daily. Two weeks after starting prednisone therapy, his aPTT had improved to $38.4 \mathrm{~s}$, factor VIII levels had increased to $10 \%$ activity and his inhibitor titer had fallen to $35.0 \mathrm{BU}$. He did not have further episodes of bleeding.

\section{Discussion}

Dupilumab is a fully humanized monoclonal antibody that inhibits interleukin (IL)-4 and IL-13 signaling by blocking the shared receptor; IL-4 $\alpha$ subunit, which in turn reduces the Th2 response [1]. It was approved by US FDA for use in moderate to severe atopic dermatitis in 2017. Three randomized, double blind, placebo controlled, multicenter trials, SOLO 1, SOLO 2 and CHRONOS evaluated the safety of dupilumab in atopic dermatitis. Adverse drug reactions reported from these trials include injection site reactions, conjunctivitis, blepharitis, oral herpes, keratitis and dry eyes $[2,3]$.

Acquired hemophilia A, a rare bleeding diathesis caused by acquired inhibitors to factor VIII, is seen in the elderly population with an incidence of 1 to 4 per million per year. It is characterized by a prolonged aPTT that is not corrected by a mixing study, reduced factor VIII levels and the presence of factor VIII inhibitor activity. It may be associated with pregnancy, autoimmune disorders, inflammatory bowel disease, solid and hematological malignancies and infections. Medications reported to cause acquired hemophilia A include penicillin, methyldopa, levodopa, interferon alpha, ampicillin, fludarabine, clopidogrel and sulfa drugs [4]. To our knowledge this is the first case of dupilumab induced acquired hemophilia A. With increasing use of dupilumab in the future, physicians should be aware of rare and serious adverse events such as acquired hemophilia A which can be treated by stopping the culprit drug, administration of recombinant factor VIIa for acute bleeding, and inhibitor eradication using immunosuppressive agents such as steroids (as in this case) or plasmapheresis.

\section{Acknowledgments}

We acknowledge the Monmouth Medical Center for allowing us access to the patient's electronic health record after obtaining patient's consent. We also acknowledge the contributions of Dr. Patrick Lee and Dr. Kenneth Granet for supervising us on this project.

\section{Financial Disclosure}

None to declare. 


\section{Conflict of Interest}

None to declare.

\section{Informed Consent}

Verbal and written consent was obtained from the patient.

\section{Author Contributions}

Dr. Dayag is the main author of this study. She initiated, conceptualized, and formulated the report and was directly involved in the care of this patient. Dr. Shaikh contributed in writing and editing the paper, and also contributed in discussion and review of literature. Dr. Patrick Lee is the hematologist on the case. He shared his office records to the writers of this report and shared his expert opinion. Dr. Granet is the primary care physician on the case. He gave updates on the outpatient status of the patient after the patient was discharged.

\section{Reference}

1. Gooderham MJ, Hong HC, Eshtiaghi P, Papp KA. Dupilumab: A review of its use in the treatment of atopic dermatitis. J Am Acad Dermatol. 2018;78(3 Suppl 1):S28-S36.

2. Simpson EL, Bieber T, Guttman-Yassky E, Beck LA, Blauvelt A, Cork MJ, Silverberg JI, et al. Two Phase 3 Trials of Dupilumab versus Placebo in Atopic Dermatitis. N Engl J Med. 2016;375(24):2335-2348.

3. Blauvelt A, de Bruin-Weller M, Gooderham M, Cather JC, Weisman J, Pariser D, Simpson EL, et al. Long-term management of moderate-to-severe atopic dermatitis with dupilumab and concomitant topical corticosteroids (LIBERTY AD CHRONOS): a 1-year, randomised, double-blinded, placebo-controlled, phase 3 trial. Lancet. 2017;389(10086):2287-2303.

4. Franchini M, Capra F, Nicolini N, Veneri D, Manzato F, Baudo F, Lippi G. Drug-induced anti-factor VIII antibodies: a systematic review. Med Sci Monit. 2007;13(4):RA55-61. 